\title{
Adjuvant Study
}

National Cancer Institute

\section{Source}

National Cancer Institute. Adjuvant Study. NCI Thesaurus. Code C15446.

A study performed secondary to and in support of a primary or base study in order to support, enhance, or expand upon its results or findings. 